\title{
Ogros y tarocafárrafas en los malecus: identidad y aliciente moral
}

\section{Ogres and tarocafárrafaes within the malecu narrative: identity and moral incentive}

\author{
Andrés Solano-Fallas ${ }^{1}$
}

Fecha de recepción: 21-1-2019

Fecha de aprobación: 7-10-2019

\begin{abstract}
Resumen
El presente artículo tiene por finalidad dilucidar cómo las figuras de los ogros (muérra maráma) y los tarocafárrafas (tarocafárrafá maráma; seres mitológicos considerados como otra especie humana ya extinta) en las pláticas de los malecus, particularmente en las Pláticas sobre ogros (Muérrajá mausírrajáca), son un medio para configurar la identidad del grupo indígena costarricense y, a su vez, cómo estas figuras fungen como aliciente moral. En lo que respecta de la configuración de la identidad, se expone en cada figura aquellos elementos que los malecus consideran ajenos y deseables a la imagen que conforman de sí. En cuanto al aliciente moral, se explica cómo los elementos en cada figura conducen a estimular ciertas prácticas morales, como también a desestimar otras conductas; comentando las respectivas consecuencias que implican en el actuar moral, dentro del contexto de la muerte, la cual es de suma importancia para los malecus.
\end{abstract}

Palabras claves: malecus, ogros (muérra maráma), tarocafárrafas (tarocafárrafá maráma), identidad, aliciente moral, muerte.

\begin{abstract}
The aim of this paper is to elucidate how the figures of the ogres (muérra maráma) and the tarocafárrafaes (tarocafárrafá maráma; mythological beings considered as another human species already extinct) within the malecu narratives, particularly the Pláticas sobre ogros (Narratives on ogres) (Muérrajá mausírrajáca), are means to shape the identity of the costarican indigenous group. At the same time, how these figures work as moral incentive. Regarding to the identity configuration, it's exposed in each figure those elements that the malecu considered external and desirable to the image of themselves. As to the moral incentive, it's explained how the elements in each figure lead to stimulate certain moral practices, as well as to reject other conducts; commenting the respective consequences that the moral acting implies, within the context of death, which is of great importance to the malecu. Key words: malecu (people), ogres (muérra maráma), tarocafárrafaes (tarocafárrafá maráma), identity, moral incentive, death.
\end{abstract}

\section{Introducción}

Los malecu(s), cuyo nombre significa "nuestra gente" (ma-, nuestro/a; lecu, gente/persona), es un pueblo indo-costarricense, perteneciente a la familia chibcha. Actualmente se encuentran en el norte del país, en el distrito de San Rafael, del cantón de Guatuso, de la provincia de Alajuela, en lo que ellos mismos denominan "Territorio
Malecu", o bien, "Reserva Indígena de los Guatusos", de acuerdo con la oficialidad estatal costarricense, según su creación en 1976. Castillo (2005a y 2005b) estima que, a mediados del siglo XIX, el territorio posiblemente alcanzaba 110000 hectáreas (1100 Km cuadrados) ${ }^{2}$, coincidiendo en gran parte con el área de la cuenca del Río Frío.

1 Licenciado en Filosofía, Bachiller en Teología. Magíster en Filosofía de la Universidad de Costa Rica. Docente en la Universidad de Costa Rica, Sede del Pacífico. Costa Rica. Correo electrónico: sadsunsea@gmail.com

2 Sobre este dato, deseo rectificar un error humano cometido en Solano-Fallas (2018: p. 3). Después de indicar las 2994 hectáreas del Territorio Malecu, reconocidas por parte del Estado costarricense, procedí a apuntar que el territorio histórico fue mucho más extenso, ya que alcanzaba las 1100 hectáreas. Claramente puede apreciarse el error, por cuanto que la segunda cifra es menor a la primera. En vez de "110o hectáreas", debe leerse correctamente "110ooo hectáreas". 
No obstante, múltiples situaciones - como el sometimiento a esclavitud y casi-exterminio por parte de los huleros nicaragüenses (1868-1900); y las posteriores migraciones internas a la zona, entre otras - provocaron la drástica pérdida de tierras. Cuando se crea la "Reserva", fue con un área de 2994 hectáreas (29.94 Km cuadrados), pero, en 1977, mediante decreto ejecutivo, se reduce la extensión a 2743 hectáreas (27.43 Km cuadrados) (Guevara-Víquez, 2011). Aunque este sea el área total del territorio, solamente 600 hectáreas (6 Km cuadrados) es "aproximadamente lo que está en control de la población indígena” (GuevaraVíquez, 2011, p. 24).

Asimismo, Castillo (2005a y 2005b) estima que, para mediados del siglo XIX, había por lo menos 17 palenques $^{3}$, y que posiblemente para esa época, la población rondaba entre las 1500 a 2000 personas. Hoy día, los palenques existentes son tres: El Sol, Margarita y Tonjibe. En lo concerniente a la población, de acuerdo con el Censo 2011 (INEC, 2013), es cercana a las 500 personas; de las cuales casi todas viven en el Territorio Malecu, junto con unas goo personas no-indígenas. Conservan su idioma, aunque no toda la población es bilingüe; además, existen variantes entre cada palenque. No obstante, se halla en un estado de decrecimiento, ya que "se encuentran adultos con una competencia únicamente receptiva en malecu o con una competencia activa reducida, niños y jóvenes con diferentes grados de competencia pasiva y productiva y ya no sobreviven sujetos monolingües en malecu" (SánchezAvendaño, 2011, p. 88).
Indicado lo anterior, este artículo explica, en primer lugar, cómo la identidad del malecu antiguo ${ }^{4}$ era conformada a partir de las figuras de los ogros (muérra maráma), así como de otro tipo de ser humano que existió antes de la transformación de la tierra, a saber, los tarocafárrafas (tarocafárrafá maráma). En segundo lugar, cómo estas dos figuras contribuían a ser un aliciente moral. Se ha escogido como obra principal las Pláticas sobre ogros, que consisten en una serie de 18 textos -originalmente transmitidos de manera oral-, que versan sobre los "incidentes ocurridos en época previa al cataclismo entre ogros y diversos grupos indígenas” (Constenla-Umaña, 2014, p. 9), principalmente los pótos quienes eran los afectados directos; los malecus quienes eran los segundos afectados, y los propios tarocafárrafas que ajusticiaron, por lo menos, a un grupo de ogros 5 .

El artículo se compone básicamente de dos apartados, con dos subpartes respectivamente. El primer apartado se centra en la figura del ogro, quien reunía aquellas características negativas o indeseables que no se consideraban propias de un malecu; el segundo, en la figura del tarocafárrafa, quien agrupaba las cualidades positivas que los malecus se atribuían a sí mismos o veían como loables de adquirir. Al inicio de cada uno, se brinda una breve descripción física de los ogros y los tarocafárrafas.

En cada primera subparte, se desarrolla cómo la figura en cuestión configura la identidad del malecu, al presentar en primer término una caracterización (lo más puntualmente

3 El término "palenque" remitía a un asentamiento multifamiliar, usualmente ubicado en la margen de algún río. Estos palenques estaban conformados por cuatro o cinco casas-ranchos grandes, a pocos metros de distancia entre cada una. En cada casa-rancho, "vivía una familia extendida compuesta por cuatro o cinco familias nucleares, cuyos miembros estaban emparentados entre sí por lazos de parentesco o matrimonio, y que en conjunto podían sumar unas 30 personas. De esta forma la población de un palenque podía oscilar entre los 90 y 120 habitantes" (Castillo, 2005a, p.80). Cada casa-rancho estaba construida sobre un plano rectangular o cuadrado, con un techo de dos vertientes, hecho de hojas de palma, y sin paredes (Castillo, 2005a, p. 80; Guevara-Berger y Chacón-Castro, 1992, p. 104).

Hoy día, el término se utiliza de manera general para referirse a las comunidades que actualmente existen en el Territorio Malecu, debido a que los lugares habitacionales dejaron de ser ranchos. En el presente son casas unifamiliares, pequeñas, de concreto o madera, y techo de zinc, a raíz de una política de vivienda del Estado costarricense a partir de 1963 (Guevara-Berger y Chacón-Castro, 1992, p. 104).

4 Sobre la diferenciación de "antigüedad” y “actualidad” de la cultura malecu, verse la Nota \#1 en Solano-Fallas (2018, pp. 19-20). Para un estudio más detallado, remitirse a Sánchez-Avendaño (2015).

5 Si bien Pláticas sobre ogros (Constenla-Umaña, 2014) es la obra principal, es relevante aclarar que en orden a comprender tanto las tramas narrativas contenidas en esta obra, como el universo malecu en general, se requiere del apoyo de otras narraciones (editadas igualmente por Constenla-Umaña) que le son vinculantes, a saber, las que corresponden a Constenla-Umaña 1992, 1993, 1996, 2011; y Constenla-Umaña e Ibarra-Rojas 2014. Además de estas, también se tomó en consideración las siguientes: Galante-Marcos (sin año, A), J.E.P Margarita-IETSAY (2000), y Mejía-Marín (1994). 
posible) de dichos seres, para que en un segundo término, por contraposición -principalmente- inferencial, pueda delimitarse cómo se concebía a sí mismo el malecu. En cuanto a las segundas subpartes, se desarrolla cómo las acciones de cada figura conducen a plantear lo que no es adecuado para el comportamiento, así como lo que efectivamente debería emularse; por lo que los ogros serían básicamente el sinónimo de lo inadecuado, mientras que los tarocafárrafas de lo aceptable, enfatizando que el actuar moral tiene consecuencias que posteriormente no se podrían esquivar, principalmente en lo relacionado a la muerte, dado que este es un tema importante en la tradición malecu. Vale finalizar con que las dos figuras funcionaban como contrapartes, tanto de la identidad como del aliciente moral, dado que uno manifiesta lo negativo, el otro lo positivo.

En lo que concierne a la manera de citar, se sigue el modo en que Constenla-Umaña editó tanto estas narraciones como otras, por cuanto permite localizar, de manera precisa y puntual, el pasaje al que se haga referencia, indirecta o directamente. Este modo consiste en que cada texto o narración está enumerado con números romanos, mientras que las líneas con números arábigos. Por ejemplo, XI, 31-33, remite al texto once, líneas 31 a la 33. Para cualquier otra obra que no remita a narraciones malecus editadas por Constenla, se utiliza el sistema solicitado de la presente revista.

\section{Contexto narrativo de las pláticas}

Los eventos que se mencionan en las pláticas, ocurren antes del famoso cataclismo, que causó la transformación de la tierra, es decir, la destrucción total de la primera creación, según se narra en el Laca Majifijica ${ }^{6}$. Se dice que los ogros (quienes no eran ni humanos ni animales) andaban de noche por la selva, mientras que de día dormían. Dado el daño que causaban a los seres humanos, principalmente a los pótos (vecinos de los malecus), y en menor medida a los mismos malecus, el dios principal, Cabecera del Nharíne ${ }^{7}$-en colaboración con su hija Jafára-, decide finalmente expulsarlos a tierras que estaban al "extremo de la gran extensión de agua, al otro lado del agua" (XVIII, 69), previas algunas masacres.

Sobre los tarocafárrafas, estos son considerados como otro tipo de ser humano, al que le fue concedida una buena muerte previa al cataclismo, porque debido a su buen comportamiento, Nharíne no quería que sufrieran las consecuencias devastadoras de la transformación de la tierra. En razón de la descripción física de estos seres, particularmente de gran altura -prácticamente gigantes-, actualmente se les puede considerar seres mitológicos, no obstante, para el imaginario malecu se trató de un linaje patrilineal que alguna vez existió (Sánchez-Avendaño, 2015, p.58, nota \#15) ${ }^{8}$.

6 Debido a que dicha obra no se ha vuelto a publicar, lo que implica que sea de difícil acceso, puede remitirse a Solano-Fallas (2016) para ver una reseña sobre los aspectos cosmológicamente relevantes.

7 Los nombres de los Dioses no revelan su verdadero nombre, sino el lugar en el que moran. Por ejemplo, este dios principal vive en la Cabecera de río Nharíne, pero por abreviación se referirá a este, y cualquier otro dios, solamente por el nombre del río en malecu.

Valga señalar que Nharíne fue el encargado de crear casi todo: en la primera vez, fue por votación unánime, ya que los demás dioses decidieron que el primero que había llegado al mundo se encargase de tal labor; en la segunda vez, fue él otra vez, porque su excompañera sentimental y madre de su hija, la diosa Aóre, le había instigado a que destruyese la humanidad, a lo cual Nharíne había finalmente aceptado, siempre y cuando, ella crease todo, pero no pudo, por lo que él tuvo que asumir la labor.

8 Parece que la existencia de los tarocafárrafas tiene un leve sustento histórico. Sánchez-Avendaño comenta que en 1783, Esteban Lorenzo de Tristán, obispo de Nicaragua y Costa Rica, comandó una fallida expedición a territorio malecu, pudiendo entrar a unos de los ríos tributarios del río Frío. En dicha expedición, “divisaron a lo lejos a tres indígenas blancos y altos” (Sánchez-Avendaño, 2015, p. 83), siendo esta la primera crónica colonial que da cuenta de malecus altos, lo cual conduce a pensar que quizá si existió, sino un linaje, por lo menos algunos indígenas malecus de estatura sobresaliente, que cesaron de existir por razones que hoy día resultan desconocidas, pero que el recuerdo colectivo los idealizó, transformándolos en los seres mitológicos que actualmente se mencionan en el texto XI.

Por otra parte, Sánchez-Avendaño expone que esta descripción cautivó la atención, no en su altura, sino en el color de su piel y pelo. En lo que resta del siglo XVIII, se reportaban que algunos malecus eran de piel blanca, rubios, o de cabello rojizo, semejante al de la guatusa, de donde supuestamente vendría el nombre de "guatusos" con que los denominaron los españoles. En el siglo XIX, básicamente se tejió la leyenda de que todos eran blancos; mientras en que la primera mitad del siglo XX se les describía de piel clara, aunque posiblemente debido al mestizaje que no estaba siendo tomando en consideración. 
Después del cataclismo, no se tiene noticia de que pasó con los ogros en las tierras a las cuales fueron exiliados, o siquiera, si los desastres naturales los alcanzaron; por su parte, el grupo de los tarocafárrafas no volvieron a ser creados, desconociéndose la razón.

\section{Los ogros (muérra maráma)}

La caracterización que ofrece las Pláticas sobre los ogros de los muérra maráma, tanto en el plano físico como accional, conducen a crear una alteridad en la que el malecu pudiera relacionarse, particularmente de manera negativa, al rechazar y despreciar todo aquello que el ogro representase. De este modo, la identidad como grupo, no se construye únicamente mediante los puntos comunes que establezcan y reproduzcan los miembros de sí mismos, sino también mediante la construcción del otro, en la que le atribuye aquellos valores que se consideren negativos de la comunidad, por ende, de sí mismos.

\subsection{Configurador de identidad}

En el caso del aspecto físico de los ogros, en modo alguno refleja rasgos humanos. En todos los textos se coincide en señalar que el ogro es de gran estatura aproximadamente el tamaño de dos personas malecu promedio-, muy velludo en todo su cuerpo; mientras que otros textos se concentran en descripciones concretas, como que la nariz es alargada, tienen varios pies que ven en varias direcciones para confundir y engañar a los seres humanos, tienen como abultamientos en su cuerpo, algunos tienen más flexiones de codos. Hay un texto en el que se les compara con los diablos (I, 65), con lo que se quiere indicar que eran bastante horribles (cf. ConstenlaUmaña, 1993, p.29), dado que los diablos no tienen figura humana; mientras que otros se limitan a expresar que "no eran poco feos” (XVI, 68). En el caso de los ogros machos, estos tienen un pene muy grande y grueso, mientras que las ogresas una vulva y pechos grandes.

En el Laca Majifijica se narra que los primeros malecu, es decir, antes del gran cataclismo, "no eran ellos/ personas de las que ahora andamos" (I, 120-121)9. No se ofrece alguna descripción posterior, salvo que las mujeres cocinaban los alimentos con el calor corporal, antes de que obtuviesen el fuego (Laca Majifijica, III), por lo que se carece de información que pueda servir para establecer un cuadro comparativo entre la fisionomía humana y la ogresca. En todo caso, la descripción física del ogro manifiesta que el malecu no se concibe a sí mismo como un ser demasiado alto y velludo, con nariz alargada, ni penes, pechos y vulvas muy grandes, ni tampoco con rasgos deformantes como los abultamientos y las flexiones de los codos, así como con los pies que ven en cada dirección. Incluso los malecus actuales ${ }^{10}$ no encajan con la descripción de un muérra, por lo que la intención de los textos estaría en ubicar al malecu -tanto al extinto por el cataclismo, como al posterior- dentro de la "normalidad". En otras palabras, en estas pláticas se utiliza al ogro como un mecanismo para establecer quién es malecu: un "quién" que excluye aquello que no le resulta compatible con la imagen de sí.

9 Aprovecho la ocasión para rectificar un error de interpretación que cometí en Solano-Fallas (2016). En la página 26, columna derecha, indiqué que los primeros malecus -quienes eran llamados "pavones" por los Dioses- no tenían rostro, haciendo referencia a las líneas 118-119 del primer texto del Laca Majifijica. Las líneas dicen lo siguiente: "Cuando inicialmente crearon los pavones/ que en su totalidad perdieron el rostro", había comprendido que la expresión de "perder el rostro” se refería a que los malecus antes del gran cataclismo no poseían cara, no obstante, me he percatado de que es una noción que remite a morir, debido a que la muerte es un concepto importante en la filosofía malecu (cf. Solano-Fallas, 2016; y Solano-Fallas y Herrera-Valenciano, 2017; J.E.P. Margarita-IETSAY, 200o, p. 48).

10 Su fisionomía habría que tomarla con reserva, debido a las mezclas culturales y fenotípicas a las que se han visto inmersos, tanto de parte de los huleros nicaragüenses (así como posteriores nicaragüenses, por encontrarse en una zona cercana a la frontera con Nicaragua), como en el avance que tuvo el Estado costarricense y la religión cristiana en la zona guatusa, y las migraciones internas de otras partes del país a Guatuso, entre otros factores.

Según Sánchez-Avendaño, en 1867 el capitán Parker -filibustero que realizó una exploración en río Frío- dio una descripción de los malecus de aquel entonces, en la que "exaltó su desarrollo muscular y su fuerza, y destacó el color cobrizo claro de su piel, el cabello negro y la carencia de adornos corporales” (Sánchez-Avendaño, 2015, p. 87). Sean las narraciones previas o posteriores a esta fecha, la descripción del muérra tampoco es compatible con los malecus divisados en ese año por Parker. 
En cuanto a las acciones "ogrescas", estas son más enfáticas en poner una línea divisoria a todo lo que no sea propio del malecu. En primer lugar, los textos insisten en que el ogro es agresivo por gusto (XVIII, 106), porque ataca sin sentido alguno a los humanos (IX, 6). El ataque que reciben los malecus, y particularmente los pótos, no corresponden a un acto de defensa propia, en el que puedan alegar que los humanos son agresores. Ni siquiera los textos sugieren que uno de sus ataques haya sido accidental. Por el contrario, los disfrutan, a tal punto que comparan a su caza humana, concretamente los pótos, con el tepezcuinte (III, 21), es decir, consideran a los pótos como simples animales.

En segundo lugar, el ogro cometía abusos sexuales. Sus principales víctimas eran mujeres pótas, aunque también había mujeres malecus que eran violadas. Algunos textos son explícitos al expresar que el ogro gozaba incansablemente de su víctima durante el día (I, 12-15; V, 5-15; VI, 16-17, 24-26; VIII, 32-33 [en todas estas referencias son malecus]), dado que este era el momento en que descansaba, mientras en la noche salía de caza o andar por la selva; mientras que, en otros, permanece implícito cuando se dice que los ogros llegaban a robarse a las mujeres (IV, 4-7; XIII, 1-2 [en estas dos referencias son malecus]; X, 16; XIV, 27; XV, 59 [en estas otras son potas]).
Además del goce inmediato forzado, que el ogro obtenía de la víctima, una intención casi secundaría consistía en engendrarles hijos para multiplicar la raza "ogresca". El embarazo inter-especista era posible según estas narraciones, pero el producto engendrado era siempre un hijo ogro (I, 24-25; V, 22; VI, 51-63 VII, 43; VIII 39; XII, 23; XIII, 44). De los textos que contiene Pláticas sobre ogros, ninguno menciona (ni siquiera sugiere) que pueda nacer un hijo humano ${ }^{11}$. Sin embargo, embarazar mujeres no parecía siempre ser el objetivo, debido a que algunas de estas violaciones terminaban en la muerte de la víctima (XI, 41), la cual no solamente sufría el trauma psicológico de ser secuestrada y violada, sino que también era devorada ${ }^{12}$.

En tercer lugar, el ogro mataba y devoraba humanos. De la serie de textos que se presentan, tres hacen alusión a que se comían a los pótos (VI, 2-7; XII, 2; y XIV, 28), no quedando claro si se refiere a pótos hombres o ambos sexo-géneros. En todo caso, los demás textos (III, 3334; XI, 41; XV, 6o; XVI, 55-56; XVII, 29; XVIII, 26, 107) especifican que se tratan de mujeres pótas, mientras que uno indica a una mujer malecu (IV, 89); por lo que sus víctimas tendían a ser pótos, y en menor medida malecus. Cabe añadir que en los textos de Pláticas de pótos, también se hace un recuento de estos hechos: en

\footnotetext{
11 Se desconoce por qué, si existen las ogresas, los ogros machos violan humanas para tratar de aumentar su raza. Quizá pueda explicarse con la característica anterior, a saber, porque era parte de su agresividad el hacerlo; además de que les gustaba cometer excesos por iniciativa propia (XIII, 10).
}

12 Lo anterior es en relación a la violación masculina ogrezca hacia mujeres, particularmente potas. En cuanto a una violación femenina ogrezca hacía un hombre es imposible, debido a que desde una razón patriarcal -que parece marcar la narración-, en el imaginario malecu el hombre "no es" violable por una hembra, ya que debería excitarlo en primer lugar para generarle una erección y luego su eyaculación, lo cual no parece posible de realizar porque las ogresas son horribles. No obstante, el que se excluya una violación de un hombre por parte de una ogresa, no elimina la posibilidad de que pueda ser embarazada por un hombre. Máxime si se tiene en cuenta que el texto VII y el XVII.

El texto VII habla de cómo un hombre mata a un ogro, porque su esposa lo estaba engañando con él: el esposo se da cuenta del engaño gracias a una lora que le cuenta lo que sucedía mientras él se iba de caza. El hombre decide matar al ogro, y cuando lo hace, su esposa "se lamentó a grandes voces" (VII, 31), lo que muestra que esta mujer desarrolló un sentimiento hacia el ogro. Este texto, si bien muestra una relación sentimental excepcional, no da pie para rechazar la idea de que lo mismo pueda suceder entre un hombre y una ogresa. Por su parte, el texto XVII (72-73), que se refiere a las hijas de lo ogros, indica que una ogresa "en efecto por sí misma bien podría tener un hijo/ sin necesidad de un ogro macho". Estas líneas no parecen remitir a que exista la posibilidad de un embarazo espontáneo o asexual, en la que no se requiera la contraparte masculina para engendrar. Sino a que podría tener un hijo proveniente de otro ser en el caso de que no haber más ogros machos, que por el contexto de la narración no puede ser otro más que un hombre, sea poto o malecu.

En otras series de textos de los malecus, a saber, Pláticas sobre felinos, antes del cataclismo se menciona que hubo vínculos sexuales, e incluso amorosos, entre humanos y felinos; empero, estas uniones inter-especistas se limitan a estas dos especies, por lo que no hay posibilidad de que un felino pueda embarazar a una ogresa. De hecho, en los textos IV y VI de Pláticas sobre ogros se menciona los tigres, pero sin establecer un vínculo entre ellos y los ogros. En el contexto del texto IV, un hombre va a matar a un ogro, y los tigres no les opusieron resistencia, ni le estorbaron en su camino, mientras que en el texto VI un tigre ayuda a una mujer a escapar del cautiverio en el que la tenía sometida un ogro; por lo que se puede inferir que no existe un vínculo inter-especista felino-ogro. De ahí que no quede más escenario posible de que sea un hombre quien pueda embarazar a una ogresa, por más remota que sea la posibilidad erótica de alcanzar una erección, consecuentemente una eyaculación. 
I, 34 y II, 15-16, 6o-62 se señala que los ogros se comían a las mujeres, mientras que III, 19 puntualiza que se comían a los pótos, sin precisar el sexo-género, por lo que puede interpretarse que se refiere a ambos. Para rematar esta agresividad, el ogro tendía a dejar las cabezas de las pótas pintadas con achiote (XI, 56-57; XV,58; XVII, 30), para que fuesen vistas por los humanos, lo cual constituye un gesto brutal simbólico de que el ogro es más fuerte.

En cuarto lugar, además de humanos, el ogro comía carne cruda (XI, 5), así como cosas inmundas, por ejemplo, los zopilotes (IX, 22-25). A pesar de que los textos no especifiquen más, por cosas inmundas se refiere a otros tipos de animales que los Dioses habían prohibido, tales como la danta adulta ${ }^{13}$, el mono congo, mono cariblanco, tigre, y sábalo real (Laca Majifijica, Texto X), entre otros más. El problema que presentaba el ogro, no era solamente comer lo inmundo, sino que lo hacía con muchas ganas, según lo manifiesta el reproche que le hace Nharíne a un ogro llamado Tío Materno del Río: "vuestros dientes se hincan en cosas inmundas" (IX, 22). Esto conduce a que el problema dietético no era que no hubiese otros animales permitidos, sino en su insistencia de conducirse mal por mero gusto, ya que comía deliberadamente lo inmundo.

En quinto lugar, el ogro mantenía relaciones sexuales incestuosas. De acuerdo con el texto IX, 19-20, si bien Nharíne le está evidenciando, al ogro Tío Materno del Río, las faltas sexuales en la que ha incurrido, su reproche no se circunscribe únicamente a este ogro en específico, sino que se generaliza, cuando expresa lo siguiente: "Perversamente... fornicáis... con... con vuestras madres,/ perversamente fornicáis con vuestras hijas". Aunque el texto solo se limite a estas dos prácticas incestuosas, no cabe excluir la condenación de otras que no son mencionadas en los textos. En todo caso, lo que interesa apuntar es que el ogro es un depravado sexual, que no solamente viola mujeres, sino que mantiene relaciones sexuales con sus parientes más próximos.
En suma, los muérra maráma cometían excesos por iniciativa propia (XIII, 10), dado que se conducían mal (IX, 13, 18), desobedeciendo los preceptos de los dioses (IX, 32). Eran agresivos sin razón, y por mero gusto; violaban mujeres; mataban y comían humanos; comían gustosamente lo inmundo; como también disfrutaban sexualmente de relaciones incestuosas. De ahí que se indique, que "no podían ser buenos" (I, 75; idea similar en IX, 17), dado que su comportamiento muestra, en un primer punto, que no eran acciones aisladas de cada ogro; en un segundo punto, de que no era uno que otro ogro aislado que las cometía, sino que era una constante.

Como se había mencionado, estas acciones ogrescas demarcan una línea divisoria entre lo que se concebía como propio de una actuación de un malecu. Las Pláticas sobre ogros fungen también como mecanismo para conformarse una identidad. El malecu no se ve a sí mismo como un ser agresivo, que buscase agredir sin ningún motivo a otra persona. Sin duda alguna, ocurrían los conflictos internos en la comunidad, pero no por una cuestión errática e impulsiva. Por ejemplo, Rigoberto Lacayo (malecu del Palenque Margarita) puntualiza que, "[c]uando había una rivalidad entre los hombres por una mujer, tenían que pelear entre los dos” (en J.E.P. Margarita-IETSAY 2000, p. 74), con palos especiales en el que cada hombre se golpeaba por turno hasta derribar al oponente, pero sin que se pretendiese matar al oponente. Puede observarse, aun cuando un conflicto se debiese a un asunto de pasiones, estas no conducían a atacar sin sentido.

El malecu tampoco se concibe a sí mismo como un agresor sexual. En el Laca Majifijica se enfatiza mucho en el respeto a la mujer. A la mujer del prójimo no se le debe molestar (XI, 1677) - entiéndase, acosar sexualmente -, como tampoco a la propia esposa, a quien no se le debe exigir sexo en demasía, puesto que, si no desea tener relaciones en la noche, no se le debe forzar (XI, 1715-1721). Tampoco a las amantes ${ }^{14}$ se les debe acosar sexualmente, ni exigírseles demasiado sexo (XI, 1678). Este respeto a la

13 En términos generales, la danta era considerada un animal inmundo, por ende, su carne prohibida para el consumo del pueblo malecu. No obstante, solo su cría estaba permitida para los ancianos (cf. Constenla-Umaña, 2014, p.111, nota \#7).

14 De acuerdo a Constela-Umaña (2014, p. 5), en los malecu se daba la poliginia. No obstante, esta práctica cambio con la influencia de la religión cristiana. 
mujer claramente no se ve en los ogros, quienes tratan a las mujeres como cosas, dado su apetito sexual violento. Cabe apuntar que dicho apetito se encuentra inferido en la descripción física del ogro. En el imaginario malecu, la enormidad de los miembros sexuales implica un carácter sexual desmedido que no respeta a la mujer. Debido a que el malecu tiene miembros sexuales "normales", no está en su caracterización la agresión hacía la mujer; además de que respeta los preceptos dados por los dioses.

Sobra señalar que no se describen como brutales, por cuanto que no mataban por gusto a otros seres humanos, ni mucho menos se comían entre sí. En este punto, es notorio resaltar que el malecu siente compasión hacía el póto que era atormentado por los ogros, por lo que desarrolla un sentimiento de solidaridad cuando -por mandato de Nharíne, su hija Jafára, y demás diosespersiguen a todos los ogros para expulsarlos del territorio poto. Según el texto XV, el malecu no solamente actuó obedeciendo una orden, sino que también se vio motivado a hacerlo por la triste y atroz situación en la que se encontraban sus vecinos (XV, 56).

Asimismo, el malecu tendía a respetar los preceptos dietéticos al no consumir carne cruda, ni mucho menos animales considerados inmundos, por lo que tampoco se asocia a sí mismo como consumidor de inmundicias.

En cuanto a lo sexual, esta parte presenta un inconveniente. Los malecus recibieron los mismos preceptos que los ogros de no mantener relaciones sexuales incestuosas. No obstante, según se narra en Laca Majifijica, los dioses decidieron castigar por segunda vez -antes de provocar el gran cataclismo- a los humanos ${ }^{15}$, particularmente a las mujeres, por mantener relaciones lésbicas e incestuosas, al enviarles tigres para que se las devoraran (Texto VI del Laca Majifijica). Luego, todos los humanos, tanto hombres como mujeres, empezaron a tener relaciones sexuales incestuosas y homosexuales; lo que causó que Nharíne finalmente atendiera la solicitud de Aóre de acabar con los humanos (Texto VII del Laca Majifijica).

Si bien la expulsión de los ogros del territorio póto fue antes del gran cataclismo, puede decirse que en un momento previo al exilio de los ogros, el malecu se diferenciaba también en este punto del ogro. Según está consignado en los textos, el malecu le achacaba al muérra sus relaciones sexuales incestuosas, particularmente con las madres y con las hijas; además, de que contrariaba abiertamente los preceptos de los tocú maráma. Lo que implicaba considerarse a sí mismo como una persona que controla sus impulsos sexuales, respeta a sus familiares, como también a sus dioses. No obstante, el propio malecu termina por sucumbir a esa práctica sexual, en un primer momento, solamente ejecutada por mujeres, en un segundo momento por toda la comunidad; lo que le hizo meritorio del tercer castigo de los dioses, a saber, el gran cataclismo donde todos los seres humanos perecieron ${ }^{16}$.

De esta manera, el malecu conforma su identidad al ponerse como espejo, no su reflejo, sino su opuesto. Físicamente, se describe como un ser "normal", ya que carece de monstruosidades y miembros excesivamente grandes; a su vez, se considera como un ser no agresivo que no mata por gusto, ni cae en canibalismo, ni mucho menos fuerza ni agrede sexualmente a las mujeres; que no come alimentos inmundos, y que mantiene -en un principio- una conducta sexual que respeta los lazos familiares. La identidad del malecu se configura, según se construye la imagen negativa del otro. Nótese que de algún modo la constitución fisionómica malecu no sugiere -según su imaginario - aspectos negativos, ni de su físico, como tampoco de su comportamiento en los planos sociales, alimenticios y sexuales, aunque termina por sucumbir nefastamente en lo último. En cambio, los rasgos del ogro evidenciaban excesos.

15 El primer castigo fue una hambruna por causas que no quedan claras en el Texto V del Laca Majifijica, en la que se especifica que no había ningún animal que comer, y que todo lo que plantaban se ponía malo. En la versión de J.E.P. Margarita-IETSAY (2000, p. 66-67) se explica que la hambruna se debió a que los malecus pretendieron tener los mismos poderes que las divinidades, por lo que un Dios -no se especifica cuál, aunque probablemente se trate de Nharíne- les prohibió la caza de animales, en especial de tortugas (ulima maráma). A diferencia del texto anterior, en esta versión si había animales, pero no los podían cazar.

16 Desde un marco general de derechos humanos, la noción que aparece de la homosexualidad en la cultura malecu antigua, es inaceptable. No solo por negar el derecho de ejercer libremente la orientación sexual de cada persona, sino también, porque la cataloga a la par de actos "perversos" e "inmorales", lo que conduce a su censura, como a la eventual muerte de la persona. 


\subsection{El ogro como aliciente moral}

En la medida en que el malecu construye un "otro" negativamente distinto a sí mismo, se pretende que las acciones del ogro enseñen cómo no debe comportarse un ser humano. La intención moral que tienen los textos es de evitar que el ser humano se comporte como un ogro, es decir, que sea agresivo, abusador sexual, asesino de otras personas, comedor de cosas inmundas, y un incestuoso degenerado. De acuerdo con los textos, un ser que actúe de esta manera, no consigue más que ser considerado por los Dioses como un "réprobo", por lo que no es de extrañar que en varias ocasiones, las pláticas caractericen al ogro de tal manera (VII, 29; VIII, 87; X, 5; XI, 35; XVIII, 119).

En la tradición malecu, un réprobo se refiere a un ser humano que se comportó inadecuadamente, cuya alma es castigada en el cielo o en alguna parte terrenal ${ }^{17}$, la cual, de vez en cuando, ronda en el mundo de los vivos; lo cual supone que existe un desprecio por parte de los dioses, dado que no le es permitido ingresar tanto a las moradas de estos últimos, como tampoco a acercarse a sus territorios. En el caso de los muérra maráma, al no ser personas ${ }^{18}$, no son castigados con ir al cielo o al río terrenal de tormentos, no obstante, el calificativo de réprobo implica que están igualmente expuestos, como los seres humanos, en primer lugar, a un rechazo enfático de parte de los dioses por su conducta; en un segundo lugar, en que son potencialmente sujetos a ser divinamente condenables. En el caso de los ogros, la condena final que recibieron fue la expulsión del territorio poto, así como su deportación al "extremo de la gran extensión de agua, al otro lado del agua” (XVIII, 69), sin posibilidad alguna de poder regresar sar a la selva, o lo que vendría a ser, sus hogares ${ }^{19}$.

17 Cuando una persona muere, habiéndose comportado mal durante su vida, pasa a sufrir castigos con los Diablos (maica maráma). Hay un diablo llamado Oronhacafá que habita en el cielo, por lo que el cielo tiene una connotación de tormento, a diferencia del paraíso cristiano. Aquí van aquellos malportados que murieron a causa de una mordedura de serpiente. Otro diablo, Jara (o Lhara), por su parte, vive en una cabecera de un río, en el mundo físico; a estos lugares van los malportados que murieron de manera trágica. Por su parte, las moradas de los tocú maráma se encuentran igualmente en el mundo físico, concretamente en las cabeceras de los ríos.

18 Las pláticas no se refieren en lo absoluto si los ogros tienen o no almas. Por el contexto, parece tratarse de un atributo único de los humanos, ya que según explica Constenla-Umaña (1993, p. 30-31), el concepto de alma remite a seres humanos.

19 Se indica que es una condena "final", por cuanto que en los textos parece que hubo varios castigos o medidas que los Dioses trataron fallidamente de ejercer, antes de tomar la solución final de exiliarlos. En un primer momento, los ogros se encontraban en el territorio malecu, no obstante, los textos IX y X mencionan que dos ogros en particular, fueron exiliados al territorio poto. En el texto IX, Nharíne le reprocha al ogro Tío Materno del Río que se conduce mal, por lo que no puede permanecer donde los malecus; motivo por el cual él será expulsado hacía la tierra de los potos, mientras que Nharíne mataba a todos los demás ogros. Por su parte, el texto X indica que el ogro Tablón de Laurel se estaba reproduciendo con ogresas, por lo que si el incremento de ogritos continuaba, sería un peligro para los malecus; de ahí que fuese exiliado por Nharíne al territorio de los potos, donde podría conjeturarse que no había ogresas.

Aunque los textos no sean explícitos en este punto, puede inferirse que las reproducciones continuaron con ogresas, dado que siguieron más ogros causando daños, ahora, principalmente sobre los potos, por encontrarse en su territorio -cuyos daños si están registrados en los textos-. En todo caso, este primer castigo, o medida, lo único que hizo fue trasladar el problema de una zona a otra, por ende, cambiar de víctima. Por lo que parece permitirse como una medida ocasional el que los seres humanos pudiesen matar a los ogros que les hacían daño (III, 55-71; IV, 19-30; XI, 42-54). De esta manera, los Dioses permitían que los propios seres humanos castigasen con la muerte a los ogros; empero no solucionaba el problema realmente, dado los constantes asedios, asesinatos y abusos sexuales por parte de los ogros hacia los potos. Por su parte, el texto XI señala que los dioses encargaron a los tarocafárrafa maráma el aniquilamiento de los ogros, en consecuencia por el agravio que cometía a los potos. No obstante, parece que solo mataron un grupo de ogros de alguna región en concreto, por cuanto que los problemas de los ogros continuaron.

Las narraciones no permiten determinar cuál solución-castigo es previo en la historia: si había matanzas motivadas por venganzas anterior a la muerte en manos de los tarocafárrafas; o si los tarocafárrafá maráma primero mataron un grupo, y los Dioses posteriormente dejaron que los demás seres humanos continuasen matando ocasionalmente; o si los dos son simultáneos, pero en zonas distintas. En todo caso, esta segunda medida o castigo no tiene éxito, debido a que los ogros continuaban atacando agresivamente sin sentido.

En razón de lo anterior, Nharíne -apoyado por su hija, Jafára, y presumiblemente por los demás Dioses- toman la decisión de exiliarlos. Sin embargo, dada la variedad de los relatos, puede conjeturarse de que no se trató de una única expulsión masiva. En un primer momento, parece tratarse de deportaciones seleccionadas, debido a que en varias ocasiones, distintos personajes trasladan a grupos de ogros al otro lado de la gran extensión de agua, y en algunas se menciona que hubo masacres, mientras que en otras no: 
Por ello, los textos buscan disuadir que una persona se comporte como un ogro, debido a que el que actúe como un ogro, no estaría actuando solamente como un réprobo, sino que estaría siendo él o ella misma un réprobo. Mientras el ogro fue castigado finalmente con exilio, previa algunas masacres -ya sea a manos de los propios dioses, o con su permiso mediante seres humanos o tarocafárrafas-, el malecu parece correr peor suerte si decide no obedecer ${ }^{20}$. Esto le haría meritorio de tener una mala muerte, que en la tradición significa que será castigado en el cielo o en el lugar terrenal de tormentos, por algún diablo, según el tipo de muerte mala que haya tenido. A su vez, implica que sus familiares borren todo recuerdo, por lo que no se trataría solamente de una muerte física, sino que, al darle muerte a su recuerdo, es como si no hubiese existido. Nótese que el castigo de los ogros se centró en un asunto que les dolía más que la propia muerte: ser arrancados de su hogar. La muerte, en sí misma, parecía no preocuparles, ya que al no ser humanos, no tienen alma, por lo que no habrá un más allá celestial o terrenal en el cual sufrir. Su sufrimiento será en vida en el exilio. Por el contrario, al malecu le preocupa la existencia post-mortem, pero lo que vaya a padecer no será por obra de los dioses -como sucedió con los ogros-, sino por los diablos. De ahí la insistencia exhortativa de que actúen según sus normas.

-En el texto I, se indica que los dioses entablaron personalmente una persecución en la que mataron a muchos ogros, ya sea asfixiándolos en sus cuevas con humo, ahogándolos, o arrojándoles agua envenenada: "Dicen, dicen que les hicieron muchas cosas malas a ellos... a los... ogros" (I, 39); hasta que Nharíne decide trasladar a los sobrevivientes en una balsa. El texto no hace referencia en quien realiza este traslado, pero parece tratarse de que son los propios Dioses quienes guían la balsa.

-En el texto VIII, por su parte, no ocurre una masacre como en el texto I. Sino que después de la muerte de un ogro que había secuestrado y violado a una mujer, Nharíne declara que se los llevarán a otro lado de la gran agua (VIII, 49). No se especifica quienes los capturaron; solamente que la tarea de trasladarlos fue encargada a los divinizados, que en la tradición malecu, son personas que por su conducta, han adquirido características "muy semejantes a las de los Dioses" (Constenla-Umaña, 1993, p. 34), y fungen como mediadores entre el dios con el cual habitan y los videntes; estos últimos que requerían igualmente de cumplir con las reglas divinas, y servían como puente de comunicación entre la gente común y los divinizados. En cuanto a los ogros, estos iban atados al cuello, por lo que les tocó nadar, mientras los divinizados eran los únicos en la balsa.

-En el texto XII se señala que los dioses encargaron la persecución a los malecus, pero guiados por los videntes de los divinizados, quienes le indicarán dónde y cómo hacer la tarea. Esta tarea incluyó una primera matanza, por lo que la deportación fue solamente de los ogros sobrevivientes. Su traslado, en esta ocasión, fue puesta a cargo de unos seres humanos, pero acompañados de los videntes. Los ogros iban con las manos atadas.

-En el texto XIII, no se indica ninguna masacre, sino que fueron capturados por los malecus, y solo uno de ellos los traslado, mientras estaban amarrados del cuello, pero en esta ocasión iban en la balsa.

Estos textos muestran que, si bien se tomó como decisión expulsar a los ogros, no se hizo pensando en una única deportación masiva. Como se puntualizó, unos incluyen masacres, y otros no. No se puede determinar el orden los hechos, a saber, cuál expulsión fue la primera; o si todas fueron simultáneas, o bien, si hubo algún orden en algunas, mientras acaecía alguna simultánea a las otras. En todo caso, parece que esta falta de planificación condujo a que no tuviese la eficacia total, debido a que en los siguientes textos se comenta una barrida general, lo que implica que todavía existían ogros.

Finalmente, parece llegarse a la condena "final". En lo que respecta a los textos del XIV al XVIII se narra que los ogros fueron capturados por los malecus, acompañados de los potos, pero con la prohibición de matarlos, bajo pena de castigo si alguien hería o mataba algún ogro. En el texto XIV se platica de la captura de los ogros machos maduros; en el texto XV de las ogresas maduras; en el texto XVI de los hijos machos de los ogros; en el texto XVII de las hijas de los ogros; y en el texto XVIII de los abuelos de los ogros. Es solamente en este último, en el que se narra que todos los ogros fueron atados de manos y puestos en la balsa, pero no se especifica quien o quienes los trasladaron. A diferencia de las expulsiones anteriores, esta parece haber sido una deportación masiva, dado su carácter sistemático y general en la captura.

20 Respecto al tema de la decisión, el malecu goza de poder elegir el no acatar las reglas divinas, a pesar de la presión que ejercen los Dioses, y las consecuencias perniciosas a las que se vería sometido si decide no hacerlo. Nharíne insiste en exhortar en que los malecus obedezcan, pero no los constriñe ni les elimina su capacidad de razonar (ver Solano-Fallas, 2016). Por ejemplo, en el texto VI, el hijo que tuvo la mujer (que había sido secuestrada por un ogro) con el hombre que la ayudó, gracias a la intervención del tigre, le es encargado de instruir a las personas diciéndoles "No... procedáis incorrectamente,/ no os conduzcáis de manera inapropiada,/ quiero... que penséis lo hagáis,/ no... os conduzcáis mal" (VI, 94-97). Obsérvese que no se les pide que obedezcan ciegamente, sino que se enfatiza en que piensen en lo que harán, por las consecuencias negativas que sus actos implicarán. El hecho de solicitarles que reflexionen sobre sus actos, es un indicador de que el malecu tiene capacidad moral deliberativa. 
Por tanto, puede afirmarse que, si bien el malecu no será exiliado en vida, ya que la expulsión no le es tan terrorífica como la mala muerte, el castigo al cual es sometido el ogro, sirve como ejemplo de que los dioses no se quedan impávidos ni ataráxicos ante el actuar de los distintos seres, según se evidencia cuando Nharíne expresa clara y puntualmente lo que pretende con estas pláticas: "Quiero que así en efecto que a todo niño de las nuevas generaciones se la contéis,/ diréis: "Así se conducían antiguamente... los réprobos de los ogros"” (XVIII, 118119). Con los ogros, intercedieron en algunos momentos de manera directa, en otros de manera indirecta, ordenando y permitiendo algunas situaciones como la venganza; con los humanos, los Dioses - después de que en el Laca Majifijica (Texto VII, 1186-1198) Nharíne decidiera no enviar nuevamente un cataclismo - no evitarían que tengan una mala muerte, ni se inmiscuirían en la manera en que los Diablos los castiguen. De este modo, aunque las consecuencias de las acciones sean distintas para los humanos, la intención moral que tienen los textos es de evitar que el ser humano se comporte como un ogro, es decir, que sea agresivo, abusador sexual, asesino de otras personas, comedor de cosas inmundas, y un incestuoso degenerado.

\section{Los tarocafárrafa maráma}

Así como existen los ogros como condensador de las cualidades negativas con las que no se reconoce un malecu, en las Pláticas sobre ogros se hace mención brevemente de otro tipo de seres humanos que cesaron de existir. Estos seres son llamados tarocafárrafa maráma, cuyo significado se desconoce actualmente. En todo caso, a ellos se les atribuyen los valores considerados positivos de cómo debería actuar un malecu, por ende, constituyen la contraparte de los ogros.

\subsection{Configurador de identidad}

Físicamente, estos seres poseen una estatura igual de grande que los ogros, pero no se ofrece luego mayor descripción. Al ser seres humanos, puede conjeturarse que su aspecto fisionómico se asemeja al del malecu, ya que no se indica que sean velludos y con partes corporales que remitan a una idea de deformidad ni monstruosidad, como tampoco con partes corporales enormes, lo cual es un indicador de que su comportamiento es radicalmente distinto al de los muérra maráma. El texto XI, al no aportar más datos, conduce a suponer que el malecu, si bien no se consideraba igual a un tarocafárrafa, por lo menos no lo concebía como un ser opuesto a sí. Es decir, su aspecto físico no se traduce en un elemento diferenciador negativo.

En cuanto al actuar del tarocafárrafa, el texto XI es muy puntual, sin ahondar en pormenores como sucede con los ogros, debido a que presupone que el oyente conoce cómo son los ogros, por lo que el narrador le deja la tarea de que realice la comparación ${ }^{21}$. Estos otros humanos "eran buenos" (XI, 2), lo cual quiere decir que no se comportaban como los ogros: no eran agresivos, ni abusadores sexuales, como tampoco asesinos de sus congéneres humanos, respetaban las reglas dietéticas al no ingerir cosas inmundas, y no eran unos degenerados sexuales, por cuanto que no mantenían relaciones sexuales con sus parientes. De ahí que se señale que era: "imposible... que se condujeran... ellos, los tarocafárrafas, como los ogros" (XI, 4).

En segundo lugar, el que no fueran como los ogros, supone que también se caracterizan por obedecer las reglas de los dioses. Ellos no solamente respetaban las normas dietéticas y sexuales, sino que también tenían un sano comportamiento con sus congéneres humanos. Además de esto, es decir, de respetar las reglas generales, los tarocafárrafas se distinguían por cumplir con satisfacción otras órdenes, que en modo alguno afectan su vida moral, alimenticia y sexual.

Cuando los ogros estaban secuestrando las esposas de los pótos, Nharíne les ordenó que los ahuyentaran de la zona, por lo que los tarocafárrafas le hicieron una trampa a un ogro, y lo arrojaron al fuego. Nharíne quedó tan complacido, según lo manifiesta cuando le dice a un tarocafárrafa (sin identificar) que: "en todo me obedeciste" (XI, 15); por lo que les vuelve a solicitar que aniquilen a todos los ogros: “todos morirán, / y allí todos perderán el

21 Debe recordarse de que se habla de "narrador" y "oyente", porque las pláticas se transmitían originalmente de manera oral. La labor de Constenla-Umaña, y su informante malecu Eustaquio Castro, consistió en ponerlas por escrito, pero conservando la narrativa oral del contenido. 
rostro" (XI, 17-18). Debido al contexto de las narraciones, el aniquilamiento de "todos" los ogros, se refiere a "todos" los ogros de una región en particular, o por lo menos de un grupo específico de ogros, ya que el problema entre los muérra maráma y los humanos, principalmente con los pótos, continuó hasta que Nharíne realizó la condena final de exiliarlos. En todo caso, la trampa fue tendida: los ogros debían cruzar un río caminando por encima de un tronco que servía de puente; los tarocafárrafas hicieron que el tronco se cayese, ahogando a "todos" los ogros. De este modo cumplieron eficaz y eficientemente con las órdenes.

La relación que se establece entre los tarocafárrafas y los malecus, como se indicó, no es opositoria, como sucedía con los ogros, empero tampoco se circunscribe al nivel de pares: ni el malecu se consideraba un tarocafárrafa, ni este podía ser llanamente igualado a un malecu. En este texto, los tarocafárrafas no sufren ningún castigo por parte de los dioses. Por el contrario, son tenidos en alta estima por su actuar. Lo que ocurre con estos otros seres humanos, consiste en servir como una especie de espejo que, en vez de reflejar la imagen del malecu de manera intacta, lo hace mostrando una versión perfeccionada, particularmente en lo que aspectos que remiten a ser bueno y obediente.

Es decir, la existencia de los tarocafárrafas en las pláticas busca que el malecu se vea a sí mismo, y adopte aquellos aspectos que ellos creen que agradan a los dioses. De este modo, el malecu se visualiza, en términos generales, a sí mismo como un ser bueno y obediente, pero perfectible; siendo los tarocafárrafas uno de sus referentes de perfección. Por ello, el recuerdo de estos seres, según se transmiten en las narraciones, tiene por objetivo que el malecu los tome como un ejemplo a seguir, lo que implica no solamente no actuar como los ogros, sino también en esforzarse en ser buenos y obedientes. Asimismo, los tarocafárrafa maráma contribuyen a reforzar aquellos puntos en los que a veces el malecu flaquea, debido a que el malecu no está predeterminado a respetar las normas, sino que es exhortado a hacerlo, por lo que tiene la posibilidad de decidir no acatarlas. Recuérdese que el cataclismo se debió por faltar a las reglas sexuales, por ende, por no comportarse de buena manera. De ahí que seres como los tarocafárrafas tengan por objetivo el ser un referente de perfección, con los cuales se identifica el malecu.

\subsection{El tarocafárrafa como aliciente moral}

En razón de lo anterior, se desprende que el tarocafárrafa tiene una función importante en ser un estímulo moral. Como se indicó, estos son referentes de perfección sobre cómo ser buenos y obedientes. Téngase en cuenta que los malecus al no regirse por principios esencialistas de "bueno y malo", no son por definición seres buenos, pero tampoco son seres malos (ver Solano-Fallas, 2016), aunque se vean a sí mismos como buenos y obedientes. Pero el aliciente moral no se reduce a que los tarocafárrafa maráma sean tomados únicamente como modelos, sino que la recompensa que reciben por parte de los Dioses, es un incentivo que puede ser atrayente para que los malecus decidan cumplir con las normas divinas.

Este incentivo consiste en tener una buena muerte. De acuerdo con Constela-Umaña, en razón de su excelente comportamiento, "Nharíne les prometió que no verían el cataclismo; en efecto, antes de que este ocurriera murieron todos de enfermedades" (2014, p.140, nota 1). Mientras los ogros fueron exiliados, así como los malecus de la primera humanidad eliminados con el cataclismo, ambos por no respetar lo que los dioses les exhortaban a cumplir; los tarocafárrafa se granjearon el aprecio de los dioses, por lo que no tuvieron que sufrir la inundación. En el imaginario malecu, morir bien se refiere a no ser mordido por una serpiente, ni morir de manera trágica, por lo que una enfermedad significa que fue una muerte natural, en modo alguna tormentosa. A su vez, esta buena muerte quiere decir que los tarocafárrafas, al ser humanos, poseen alma, la cual pasará habitar en las casas de los dioses.

De esta manera, el premio que reciben estos otros seres humanos, es un aliciente para que el malecu cambie su forma de actuar, en caso de que esté desobedeciendo, o bien, modifique alguno que otro elemento que pueda poner en peligro su buena muerte, como también a que se esfuerce constantemente en ser bueno y obediente, es decir, en cumplir plenamente con lo que se identifica. 


\section{Conclusión}

Puede apreciarse que cada figura cumplía una importante función en la conformación de la identidad del malecu antiguo, a su vez, servían como alicientes morales. Como resume explicativamente Sánchez-Avendaño, desde un enfoque constructivista, "las identidades proporcionan los recursos para moldear las subjetividades y las experiencias de cada sujeto" (2015, p. 16). El malecu plasmó en estas narrativas, la manera en cómo se concebía a sí mismo, agrupando por un lado los aspectos negativos que rechazaba o no deseaba, a pesar de sucumbir en algunos -como las faltas sexuales-; por otro, concentrando aquellos puntos positivos que, si bien no se veía como pleno cumplidor, por lo menos se encaminaba hacia ellos al haberlos idealizado como deseables.

Esta imagen que se construye de sí, no es un asunto meramente descriptivo en el que se buscase especificar con mayor o menor detalles aquellos rasgos que valoraban y despreciaban como propios o inadecuados de un malecu, ni tampoco es un asunto que se limite a lo adscriptivo, en el tanto que solamente sirviese para crear un marco referencial de pertenencia grupal. Sin duda alguna, el ogro y el tarocafárrafa presentan una identidad grupal (o social), en la que no se ofrece pormenores individuales de cada malecu, sino en lo que se comparte, en lo que es común -o se supone y proyecta como tal- (cf. Sánchez-Avendaño, 2015, p. 21), pero sin confinarse a esto.

La identidad que se configura, desde los ogros y los tarocafárrafas, es relevante para ofrecer los recursos morales de actuación, dado que proporcionan en sí mismos alicientes morales que son de capital importancia para el malecu antiguo. Estas dos figuras muestran al malecu que, en tanto malecu que es, debe haber una correspondencia en sus acciones que le permitan identificarse como tal. Por ello la insistencia de enfatizar en los ogros lo negativo con lo que no quería relacionarse tajantemente, y que, aun cuando el castigo de los ogros fue distinto, pone en claro que un comportamiento "ogresco" o similar no es adecuado para alguien que se identifique como malecu. De manera equivalente sucede con los tarocafárrafas quienes sirven para idealizar una conducta deseable y adecuada de un malecu, dada la recompensa que obtuvieron por sus acciones.
Por consiguiente, entre identidad y aliciente moral, desde las figuras del ogro y el tarocafárrafa, existe una correlación bidireccional en la que la primera conforma el campo de la autoimagen, implicando la realización o desestimación de ciertas acciones, mientras que, en la segunda, permite juzgar si hay o no una correspondencia entre lo que se hace y lo que se dice ser.

\section{Referencias bibliográficas}

Castillo, R. (2005a). "El territorio histórico maleku de Costa Rica”. Revista Reflexiones, 84 (1), p.71-85.

Castillo, R. (2005b). "Población indígena maleku en Costa Rica”. Anuario de Estudios Centroamericanos, 31, p.115-136.

Constenla-Umaña, A. (1992). "Hagiografía y Antihagiografía en la tradición oral guatusa" en Filología y Lingüística, XVIII, 1, p.83-124.

Constenla-Umaña, A. (1993). Laca Majifijica. La Transformación de la Tierra. (Introducción, transcripción y traducción de Adolfo ConstenlaUmaña; narración por Eustaquio Castro y Antonio Blanco). San José: EUCR.

Constenla-Umaña, A. (1996). Poesía tradicional indígena costarricense. (Serie antológica). San José: EUCR.

Constenla-Umaña, A. (2011). Pláticas sobre felinos. (Narración de Eustaquio Castro; introducción, transcripción y traducción de Adolfo ConstenlaUmaña). San José: EUCR.

Constenla-Umaña, A. (2014). Pláticas sobre ogros. (Narración y colaboración en la transcripción y traducción de Eustaquio Castro; introducción, transcripción y traducción de Adolfo ConstenlaUmaña). San José: EUCR. 
Constenla-Umaña, A.; e Ibarra-Rojas, E. (2014). "Anotaciones etnohistóricas sobre los indígenas botos: confluencia de datos históricos, antropológicos y de la tradición oral malecu" en Estudios de Lingüística Chibcha, 33, p. 111-164. [Este artículo contiene las 'Pláticas de bótos', en su versión malecu y española]

Galante-Marcos, E. (directora). (sin año, A): Malecu Usirraca Marama. Libro de Leyendas Malecu. Proyecto Rio Frío. (Carece de editorial e imprenta, y de lugar)

Guevara-Berger, M.; y Chacón-Castro, R. (1992). Territorios indios en Costa Rica: orígenes, situación actualy perspectivas. San José: García Hermanos.

Guevara-Víquez, F. (2011). Cronología básica de los pueblos indígenas de Costa Rica: desde los inicios del siglo XVI hasta el año 20oo. San José: Siwá Pákö [Comisión integrada por MEP (Ministerio de Educación Pública) - UCR (Universidad de Costa Rica) - UNA (Universidad Nacional de Costa Rica) - UNED (Universidad Estatal a Distancia)]

Instituto Nacional de Estadística y Censos (INEC) (Costa Rica) (2013). X Censo Nacional de Población y VI de Vivencia 2011. Territorios indígenas: principales indicadores demográficos y socioeconómicos. San José: INEC.

Junta de Educación Palenque Margarita (J.E.P. Margarita) Instituto de Estudios de las Tradiciones Sagradas de Abia Yala (IETSAY). (200o). Narraciones malekus. San José: Tikal.

Mejía-Marín, N. (1994). Historias malecus. (Narración y traducción de Noemy Mejía-Marín; introducción y recopilación de Raúl Bolaños Arce, Juan de Dios Ramírez Gatgens, y Rocío Alvarado Cruz). Heredia: EUNA.

Sánchez-Avendaño, C. (2015). La cola de la iguana. El pueblo malecu ante el desplazamiento de su lengua y su cultura tradicional. San José: EUCR.
Sánchez-Avendaño, C. (2011). "Caracterización cualitativa de la situación sociolingüística del pueblo malecu” en Estudios de Lingüística Chibcha, 30, p. 63-90.

Solano-Fallas, A. (2016). "El bien y el mal, la elección, y la muerte en la cosmogonía malecu "Laca Majifijica"/ The Good and Evil, the Chossing and the Death in the Cosmogony Malecu "Laca Majifijica”" en Revista Pensamiento Actual, Vol. 16, No. 26, p.25-32.

Solano-Fallas, A. (2018). "Ogros en los malecus: sobre la elección y la responsabilidad en la filosofía antigua malecu" en Revista humanidades, Vol. 8, No. 2, p.1-29.

Solano-Fallas, A.; y Herrera-Valenciano, M. (2017). "Felinos en los malecus: simbolización de la muerte como aliciente moral" en Revista de Filosofía de la Universidad de Costa Rica, Vol. LVI, No. 144, Enero-Abril, p. 171-178.

\section{Literatura consultada}

Bolaños-Esquivel, B.; y González-Campos, G. (2010). Las miradas con que vemos. Análisis de la representación audiovisual de los pueblos indígenas de Costa Rica. San José: Vicerrectoría de Acción Social, Universidad de Costa Rica.

Echánove-Trujillo, C. (1976). Diccionario de sociología. México: Jus.

Espinoza-Romero, E.; Mejía-Marín, N.; y OvaresBarquero, S. (2011). "El Malecu: Una cultura en peligro de extinción. Maleku: A culture in Danger of Extintion." Revista Electrónica Educare. Vol. $\mathrm{XV}, \mathrm{N}^{\circ}$ Extraordinario, [69-84], Octubre.

Fundación Coordinación de Pastoral Aborígen (FUNCOOPA)-Instituto de Estudios de las Tradiciones Sagradas de Abia Yala (IETSAY). (1999): Pueblos indígenas de Costa Rica: historia y situación actual. San José: Tikal. 
Galante-Marcos, E. (directora). (sin año, B): Diccionario Malecu. Proyecto Rio Frío. (Carece de editorial e imprenta, y de lugar)

Ibarra-Rojas, E. (2011). "Los nicaraos, los indios votos y los huetares en escenarios conflictivos en el siglo XVI" en Cuadernos de Antropología, No. 21, p.1-23.

Kottak, C. P. (2006). Antropología cultural. Madrid: McGraw-Hill.

Maalouf, A. (2002). Identidades Asesinas. Madrid: Alianza.

Margery-Peña, E. (2007). Estudios de mitología comparada indoamericana. Tomo II. El origen del fuego y concepciones sobre el lugar de los muertos en pueblos indoamericanos. San José: EUCR.

Salazar-Salvatierra, R. (2006). El indígena costarricense: una visión etnográfica. The Costa Rican indigenous people: an ethnographic overview. Cartago: Editorial Tecnológica de Costa Rica.

Sánchez-Avendaño, C. (2013). "Apropiación por parte de los miembros del pueblo malecu de la ortografía práctica de su lengua" en Estudios de Lingüística Chibcha, 32, p. 209-229.

Sen, A. (2007). Identidad y Violencia. La ilusión del destino. Buenos Aires: Katz.

Solano-Salazar, E. (2004). "La población indígena en Costa Rica según el censo 2000” en Rosero Bixby, L. (ed.). Costa Rica a la luz del censo del 20oo. San José: Centro Centroamericano de Población de la Universidad de Costa Rica.

Tenorio-Alfaro, L. (1990). Reservas indígenas de Costa Rica. San José: Imprenta Nacional.

Theodorson, G.; y Theordorson, A. (1978). Diccionario de sociología. Buenos Aires: Paidós.
Zavala, M.; y Araya, S. (2008). Literaturas indígenas de Centroamérica (Segunda edición revisada). Heredia: EUNA. 